\title{
THE TRAVELING SALESMAN PROBLEM AND HARMONIC ANALYSIS
}

\author{
PETER W. JONES
}

In this paper we propose to discuss some relationships between the classical Traveling Salesman Problem (TSP), Littlewood-Paley theory, and harmonic measure. This circle of ideas is also closely related to the theory of Cauchy integrals on Lipschitz graphs, and this aspect is discussed more fully in the paper of David and Semmes [2] in these proceedings. The main differences between the subjects of [2] and this paper are that the results here are valid for one dimensional sets, whereas [2] treats $d$ dimensional sets. The sets considered in [2] are required to satisfy the natural thickness condition of David-type, while the sets considered here have no such restriction. The reason David and Semmes have such a restriction is their interest in boundedness of singular integrals. While we are also interested in this problem and the related problem of analytic capacity, singular integrals do not play quite as strong a roble in the topics discussed here.

Let us begin by recalling the classical TSP. Let $K=\left\{x_{1}, \ldots, x_{n}\right\}$ be a finite collection of points in $\mathbb{R}^{2}$. Find $\Gamma_{h}$, the shortest Hamiltonian cycle passing through $K$, and let $l\left(\Gamma_{k}\right)$ denote its arclength. This is a "hard" problem, and it seems unlikely that we will ever sec a fast algorithm for solving it. On the other hand, consider the problem of finding the shortest connected set $\Gamma_{0}$ which contains $K$. Then fast algorithms exist for computing $\Gamma_{0}$, and since it is casily shown that

$$
l\left(\Gamma_{h}\right) \leq 2 l\left(\Gamma_{0}\right)
$$

the harmonic analyst who does not consider constants to be important might consider the finite TSP to be solved. (See [7] for more information on the classical TSP.)

In whatever form it is posed, the classical TSP may seem unnatural to harmonic analysts because of the restriction that $K$ is finite. We consider instead general sets $K$ and pose the following question: can we find, up to a constant multiple, the length of the shortest curve which passes through $K$ ? Recall that a curve $\Gamma$ is called rectifiable if $\Gamma=F([0, A])$ for some $A<\infty$ where $F$ is Lipschitz, $|F(x)-F(y)| \leq|x-y|$. It is not too hard to see that $\Gamma$ is a rectifiable curve if and only if $\Gamma$ is connected and the one dimensional Hausdorff measure of $\Gamma$ is finite, $l(\Gamma)<\infty$. A restatement of our problem is thus the following: characterize subsets of rectifiable curves. It is perhaps surprising that this problem has a rather simple answer in terms of a geometric Littewood-Paley type 
theory. Such an approach was first presented for the special case of Lipschitz curves in the Proceedings of the previous El Escorial meeting [5]. The point of that argument was to give another proof of boundedness of Cauchy integrals by examining geometric square functions.

If $K \subset L$ where $L$ is a line, our problem is easily solved. We therefore should attempt to see how much $K$ deviates from lines at all points of $K$ and at all scales. We define for a dyadic square $Q$ the quantity

$$
\beta_{K}(Q)=\inf _{L} \sup _{z \in K \cap 3 Q} l(Q)^{-1} \text { distance }(z, L)
$$

where $l(Q)=2^{-n}$ is the sidelength of $Q$ and where $3 Q$ is the square with same center as $Q$ and $l(3 Q)=3 l(Q)$ (the infimum is taken over all lines $L$.) Then it turns out that $K \subset \Gamma, \Gamma$ a rectifable curve if and only if $K$ is bounded and

$$
\beta^{2}(K) \equiv \sum_{Q} \beta_{K}^{2}(Q) l(Q)<\infty,
$$

where the sum is taken over all dyadic $Q$. We remark that $\beta^{2}(K)$ is easily seen to be equivalent to

$$
\int_{0}^{\infty} \int_{\mathbb{R}^{2}} \beta_{K}^{2}(z, r) r^{-2} d x d y d r
$$

where $\beta_{K}(z, r)$ is defined using the disk $\{\varphi:|\varphi-z| \leq r\}$ instead of a square $Q$. The reason for choosing our definition is purely psychological; it is helpful when counting Whitney squares. Let $\Gamma_{0}$ denote the shortest curve containing $K$.

Theorem 1. [6] There is a universal constant $C_{0}$ such that for all $K$,

$$
C_{0}^{-1} \leq \frac{\text { diameter }(K)+\beta^{2}(K)}{l\left(\Gamma_{0}\right)} \leq C_{0} .
$$

I have been informed by S.Y. Chang and J. Garnett that their student K. Okikiolu has extended Theorem 1 to be valid in $\mathbf{R}^{n}, n \geq 3$. This involves finding a different proof of the second inequality for arbitrary curves $\Gamma_{0}$.

The first inplication of Theorem 1 is obtaincd by giving a direct construction of a curve $\Gamma$ such that $l(\Gamma) \leq C_{0}\left(\operatorname{diam}(K)+\beta^{2}(K)\right)$. Without being too specific, our method is closely related to the following algorithm. Suppose $K$ is closed, and suppose curves $\Gamma_{1}, \Gamma_{2}, \ldots, \Gamma_{n}$ have been constructed, such that $\Gamma_{k}$ consists of $k$ line segments $I_{j}^{k}$ with endpoints on $K$. Let $x_{n+1} \in K$ have maximal distance to the collection of all endpoints of the intervals $I_{j}^{n}$. Now replace in the most efficient manner one of the intervals $I_{j}^{n}$ by two intervals so as to obtain a new curve $\Gamma_{n+1}$ which also contains $x_{n+1}$. As an easy example suppose $K$ consists of three points, $K=\{0,1,1 / 2+i \delta\}$ where $0<\delta<1$. Then clearly 
$\Gamma_{0}=[0,1 / 2+i \delta] \cup[1 / 2+i \delta, 1]$. Take $\Gamma_{1}=[0,1]$ so that $l\left(\Gamma_{1}\right)=$ diameter $K$. Then the algorithm gives $\Gamma_{2}$ as our desired $\Gamma_{0}$. Also notice that

$$
\begin{aligned}
l\left(\Gamma_{2}\right) & =l\left(\Gamma_{1}\right)+\left(l\left(\Gamma_{2}\right)-l\left(\Gamma_{1}\right)\right) \\
& =\operatorname{diameter}(K)+\left(\sqrt{1+4 \delta^{2}}-1\right) \\
& \sim \operatorname{diameter}(K)+\delta^{2} \\
& \sim \operatorname{diameter}(K)+\beta_{K}^{2}\left([0,1]^{2}\right) l\left([0,1]^{2}\right) .
\end{aligned}
$$

For more general $K$, a variant of this algorithm works (essentially) because for the constructed curves $\Gamma_{n}, l\left(\Gamma_{n+1}\right)-l\left(\Gamma_{n}\right) \leq C \beta_{K}^{2}\left(Q_{n}\right) l\left(Q_{n}\right)$, and each $Q_{n}$ is counted only a finite number of times. Thus setting $\Gamma=\lim \Gamma_{n}$ (assuming that makes sense), $l(\Gamma) \leq l\left(\Gamma_{\mathrm{t}}\right)+\sum_{n=1}^{\infty}\left(l\left(\Gamma_{n+1}\right)-l\left(\Gamma_{n}\right)\right) \leq$ diameter $(K)+$ $C \sum_{Q} \beta_{K}^{2}(Q) l(Q)$. This is in any case the idea we use.

The proof of the second inequality in Theorem 1 uses a different idea. One first notices that the result follows from a careful application of the Pythagorean theorem if $K$ is the boundary of a Lipschitz domain. One then uses the following result.

Theorem 2. Suppose $\Omega$ is a simply connected domain. Then

$$
\Omega=\left(\bigcup_{j} \bar{\Omega}_{j}\right) \cap \Omega
$$

where $\left\{\Omega_{j}\right\}$ is a disjoint collection of $C_{0}$ Lipschitz domains with the property that each $\Omega_{j} \subset \Omega$ and each $z \in \Omega$ is in no more than $C_{0}$ of the sets $\bar{\Omega}_{j}$. Furthermote,

$$
\sum_{j} l\left(\partial \Omega_{j}\right) \leq C_{0} l(\partial \Omega)
$$

See [4] where this is extended to minimal 2 surfaces. One now completes the proof of Theorem 1 by using Theorem 2 and the fact that the result is true for Lipschitz curves. To be a little more precise, take a curve $\Gamma$ and enlarge it by Theorem 2 to a curve $\Gamma^{\prime}, l\left(\Gamma^{\prime}\right) \leq C_{o} l(\Gamma)$, such that $C \backslash \Gamma^{\prime}=U \Omega$, is a union of Lipschitz domains plus a disk at $\infty$. If $\beta,(Q)$ is the " $\beta$ " computed for $\partial \Omega_{3}$ and $Q$,

$$
\sum_{Q} \beta_{j}^{2}(Q) l(Q) \leq C l\left(\partial \Omega_{j}\right)
$$

When one computes $\beta$ 's for $\Gamma^{\prime}$, the curves $\partial \Omega_{j}$ essentially act independently so that (morally)

$$
\beta_{\Gamma}^{2}(Q) \leq \beta_{\Gamma^{\prime}}^{2} \leq \sum_{j} \beta_{j}^{2}(Q)
$$


Some minor adjustments are of course needed to make this idea work. In any case, one ends up with the estimate

$$
\begin{aligned}
\beta^{2}(\Gamma) \equiv \sum_{Q} \beta_{\Gamma}^{2}(Q) l(Q) & \leq C \sum_{j, Q} \beta_{j}^{2}(Q) l(Q) \\
& \leq C^{2} \sum_{j} l\left(\partial \Omega_{j}\right) \\
& \leq 2 C^{2} l\left(\Gamma^{\prime}\right) \leq 2 C^{3} l(\Gamma) .
\end{aligned}
$$

We mention an amusing corollary to Theorem 2:

If $\Gamma \subset \mathbf{R}^{2}$ is connected, there is a connected set $\Gamma^{\prime}$ such that $l\left(\Gamma^{\prime}\right) \leq C_{0} l(\Gamma)$ and such that any points $x, y \in \Gamma^{\prime}$ can be connected by an arc $\gamma \subset \Gamma^{\prime}$ with $l(\gamma) \leq 3|x-y|$.

We offer a reward of $\$ 100$ to anyone who provides a proof of this result which does not use $L^{2}$ estimates. (Admittedly, this is not precisely defined.)

In [1] Christopher Bishop and this author use Theorem 1 to deduce some new results on harmonic measure. The main result proved there is the following.

Theorem 3. Suppose $\Omega$ is simply connected and $z_{0} \in \Omega$ satisfies distance $\left(z_{0}, \partial \Omega\right)=1$. Then if $\Gamma$ is any rectifiable curve, and $E \subset \Gamma \cap \partial \Omega$,

$$
\omega_{z_{0}}(E) \leq \delta(l(E), l(\partial \Omega)) \text {. }
$$

Here $\omega_{z_{0}}$ denotes harmonic measure for $\Omega$ mcasured at $z_{0}$, and for $l(\partial \Omega) \leq A$,

$$
\delta(t, l(\partial \Omega)) \leq \delta(t, A) \underset{t \rightarrow 0}{\longrightarrow} 0 .
$$

The prototypical such result is the classical form of the F.M. Riesz theorem due to Laurentiev: when $\Gamma=\partial \Omega$ and $E \subset \Gamma$,

$$
\omega_{z_{0}}(E) \leq \frac{C_{0} \log (l(\Gamma))}{|\log (l(E))|+1} .
$$

Theorem 3 had been conjectured by $\emptyset$ ksendal, who proved it in the case where $l(E)=0$ and $\Gamma=\boldsymbol{R}$. Later results for special curves $\Gamma$ are due to Kaufman, and Wu. See [1] for a more complete history. A rather easy corollary to Theorem 3 is

Theorem 4. For a curve $\Gamma$,

$$
\sup _{\Omega, \Phi} l\left(\Phi^{-1}(\Gamma \cap \Omega)\right)<\infty
$$

if and only if

$$
\sup _{z, r} r^{-1} l(\{\zeta \in \Gamma:|z-\zeta| \leq r\})<\infty
$$


Here the first supremum is taken over all simply connected $\Omega$ and all Riemann mappings $\Phi$ from the unit disk to $\Omega$. The curves satisfying the second inequa]ity of the theorem are those satisfying the Guy David (or "Ahlfors-David") condition; they were shown by David to be exactly the curves for which the Cauchy integral is $L^{2}$ bounded. The first conclusion of Theorem 4 was first proven by Hayman and Wu, and a little latter by Garnett, Gebring, and Jones, when one has $\Gamma=\mathbb{R}$. This was extended to chord arc curves $\Gamma$ by Fernández and Hamilton. Again, see [1] for a more detailed history.

The proof of Theorem 3 is rather long and complicated, but the main idea can be rather easily explained. Let $\Omega, \Gamma$ be fixed and suppose $E \subset \Gamma \cap \partial \Omega$ satisfies

$$
\omega_{z_{0}}(E) \geq \varepsilon_{0} .
$$

Let $\Phi: \mathbb{D} \rightarrow \mathbb{C} \backslash E$ be the universal covering map (we may assume $E$ is closed), and let $\Phi(0)=z_{0}$. Setting $D=\Phi^{-1}(\Omega)$ (let $0 \in D$ to make this well defined), we see by the maximum principle that $l(\partial D \cap \boldsymbol{J}) \geq 2 \pi \varepsilon_{0}$. A theorem due to Pommerenke [8] allows one to conclude that

$$
l(\partial T \cap \mathbb{\nabla}) \geq 2 \pi \varepsilon_{0},
$$

where $\mathfrak{T}$ is the normal fundamental domain associated to $\Phi$ and $\mathbb{C} \backslash E$. Since $T$ has boundary consisting of a subset of $\mathbb{T}$ plus some circular arcs orthogonal to $\mathbb{E}, l(\partial T) \leq \pi^{2}$, and the harmonic measure in $\mathfrak{T}$ (measured at the origin) of $\pi \cap \partial \mathfrak{T}$ is greater than $\varepsilon_{1}=\varepsilon_{1}\left(\varepsilon_{0}\right)$. We may thus assume that

$$
\Omega=\Phi(\mathfrak{T}) \text {. }
$$

If it were the case that $l(\partial(\Phi(T)))<\infty$, one could simply invoke the F.M. Riesz-Laurentiev theorem to obtain Theorem 3 . This turns out to be false, but a closely related result is true. Let $D$ be a Lipschitz domain, $D \subset \mathfrak{T}$ and for every $z \in D$,

$$
\omega_{z, D}(\mathbb{\nabla} \cap \partial D) \geq \varepsilon_{2} .
$$

Then it turns out that

$$
l(\partial(\Phi(D))) \leq C\left(\varepsilon_{2}\right),
$$

and this establishes Theorem 3. The difficult part of the argument is to prove the last inequality. To this end set $\Phi^{\prime}(z)=e^{\varphi(z)}$ and define $d(z)=$ distance $(z, \partial \mathcal{T})$. Then the now well known $L^{2}$ theory applied to $\left(\Phi^{\prime}\right)^{3 / 2}$ tells us

$$
l(\partial \Phi(D)) \sim\left|\Phi^{\prime}(0)\right|+\iint_{D}\left|\Phi^{\prime}(z) \| \varphi^{\prime}(z)\right|^{2} d(z) d z d y .
$$

Applying the $L^{2}$ inequality once more yields

$$
l(\partial \Phi(D)) \sim\left|\Phi^{\prime}(0)\right|+\left|\Phi^{\prime \prime}(0)\right|+\iint_{D}\left|\Phi^{\prime}(z)\right|\left|\varphi^{\prime \prime}(z)+\frac{1}{2}\left(\varphi^{\prime}(z)\right)^{2}\right|^{2} d(z)^{3} d x d y .
$$


The first two terms are "trivial", and we are left with the task of bounding the last integral. For reasons to be explained we now change the plus sign in the integral to a minus sign, and calling the integral I, we obtain

$$
\begin{gathered}
I=\iint_{D}\left|\Phi^{\prime}(z)\right|\left|\varphi^{\prime \prime}(z)-\frac{1}{2}\left(\varphi^{t}(z)\right)^{2}\right|^{2} d(z)^{3} d x d y \\
+ \text { "Other term". }
\end{gathered}
$$

It turns out that the two terms above look quite different and require different arguments. We will not explain how to bound the "Other Term", and will concentrate on the first. A surprising fact is that although the mapping

$$
\varphi^{\prime \prime}(z)+1 / 2\left(\varphi^{\prime}(z)\right)^{2} \longrightarrow \varphi^{\prime \prime}(z)-1 / 2\left(\varphi^{\prime}(z)\right)^{2}
$$

is seriously nonlinear, there is none the less an $L^{2}$ theory (at least for "nearly" univalent $\Phi$ ) for the mapping

$$
\left(\Phi^{\prime}(z)\right)^{1 / 2}\left(\varphi^{\prime \prime}(z)+1 / 2\left(\varphi^{\prime}(z)\right)^{2}\right) \longrightarrow\left(\Phi^{\prime}(z)\right)^{1 / 2}\left(\varphi^{\prime \prime}(z)-1 / 2\left(\varphi^{\prime}(z)\right)^{2}\right) .
$$

Why have we changed the plus sign to a minus? The answer is that

$$
\begin{aligned}
\varphi^{\prime \prime}-1 / 2\left(\varphi^{\prime}\right)^{2} & =\left(\frac{\Phi^{\prime \prime}}{\Phi^{\prime}}\right)^{\prime}-\frac{1}{2}\left(\frac{\Phi^{\prime \prime}}{\Phi^{\prime}}\right)^{2} \\
& =S \Phi
\end{aligned}
$$

is the Schwarzian of $\Phi$, and $S \Phi$ is related to geometry! In particular $S \Phi \equiv 0$ when $\Phi$ is a Möbius transformation. Now our $\Phi$ is associated to $E$, a subset of a rectifiable curve, and by Theorem 1, $E$ looks "flat most of the time". In particular, if $z \in D$, the condition

$$
\omega(\Phi(z), E, \Omega) \geq \epsilon
$$

implies that there must be a rather large amount of $E$ (in some sense) at $\Phi(z)$, on scale

$$
\text { distance }(\Phi(z), E) \sim\left|\Phi^{\prime}(z)\right| d(z) .
$$

(This relation " $\sim$ " is just the Koebe $1 / 4$ theorem plus our choice of the definition of $D$ ). Now since $E$ is "mostiy flat" and "dense near $\Phi(z)$ " we morally have that $E \approx$ straight line near $\Phi(z)$. This would force $S \Phi(z) \approx 0$ because $\Phi$ would be nearly a Möbius transformation near $z$.

We now attempt to quantify the above intuition. Let $R \subset \mathfrak{T}$ be a square in the Whitney decomposition of $T$, and assume that $R \cap D \neq \phi$. Then $\Phi(R)$ has diameter $\sim\left|\Phi^{\prime}(z)\right| d(z) \sim$ distance $(\Phi(R), E)$. Let $Q$ be a dyadic square, 
$\Phi(R) \cap Q \neq \phi, l(Q) \sim A$ diameter $(\Phi(R))$ where $A$ is a large fixed constant. Then while we cannot expect $S \Phi \equiv 0$ on $R$, it turns out that

$$
\begin{aligned}
& |S \Phi(z)| d(z)^{2} \leq C_{\theta} \beta_{E}(Q) \\
& \text { t"Higher Order Term" }
\end{aligned}
$$

on all of $R$. Ignoring the "Higher Order Term" we see that essentially

$$
\iint_{R}\left|\Phi^{\prime}(z)\right||S \Phi(z)|^{2} d(z)^{3} d x d y \leq C_{0} \beta_{E}^{2}(Q) l(Q)
$$

and summing over all $R$ we find that "to first order",

$$
\begin{aligned}
& \iint_{D}\left|\Phi^{\prime}(z)\right||S \Phi|^{2} d(z)^{3} d x d y \\
& \leq C_{0} \sum_{Q} \beta_{E}^{2}(Q) l(Q) \\
& \leq C_{1} l(\Gamma) .
\end{aligned}
$$

While our reasoning was a bit imprecise, the last inequality is actually true. As we remarked earlier, it is unfortunate that at this point in time we know of no elementary arguments to prove Theorem 3 .

We now attempt to explain our mysterious remark that the so-called "Other Term" can be ignored in the proof of Theorem 3. Let $L$ be a Lipschitz domain and again set $d(z)=$ distance $(z, \partial L)$. The following, as yet unpublished result of Bishop and the author was proven only in 1990.

Theorem 5. Suppose $\Phi$ is univalent on $L$ and

$$
\iint_{L}\left|\Phi^{\prime}(z)\right||S \Phi(z)|^{2} d(z)^{3} d x d y \equiv B<\infty,
$$

Then $\Phi^{\prime} \in H^{1 / 2-\varepsilon}(L)$ with norm depending only on $\Phi^{\prime}$ (center of $L$ ), $B, \varepsilon$, and the Lipschitz constant of $\partial L$.

Notice that the space $H^{1 / 2-\varepsilon}$ is sharp: consider a Möbius transformation. The assumption that $\Phi$ is univalent can be relaxed to the condition that $\varphi$ is a Bloch function, but this is not fundamentally different. To prove Theorem 3 , we use the fact, as outlined above, that the integral in Theorem 5 is bounded. Then we simply apply Jensen's inequality. An unfortunate fact of our present proof of Theorem 5 is that it is very similar to our previous proof that the so-called "Other Term" is bounded. (We do, however, avoid a rather delicate geometric argument which was used for "Other Term".)

Finally, we wish to close with some cryptic comments. In [5], the boundedness of the Cauchy integral was (re) studied by essentially showing that the 
Cauchy integral operator on a Lipschitz curve could be written as a sum of a "diagonal" operator (the Hilbert transform) and an "off diagonal" operator (a paraproduct). While this philosophy was by no means new, the novelty was that the paraproduct corresponded to the number $\beta^{2}(\Gamma)$ in a very well defined manner. In a very real sense, Theorem 1 provides a way to associate a paraproduct to any planar set. This imprecise statement has been extensively developed by David and Semmes [3]. The study of $\beta^{2}(K)$ and the Schwarzian, $S \Phi$, of a universal covering map are "the same" via Theorem 2 and the proof of Theorem 3. Furthermore, the Schwarzian corresponds to a certain nonlinear paraproduct via Thcorem 5. All objects mentioncd here are nonlinear Littlewood-Paley expressions. The miracle is that, as first shown for Cauchy integrals, the nonlinearity does not destroy the $L^{2}$ theory. One can loosely explain this by saying that the nonlinear effects are controlled by $L^{2}$ bounds on geometry. We are left with the following diagram:

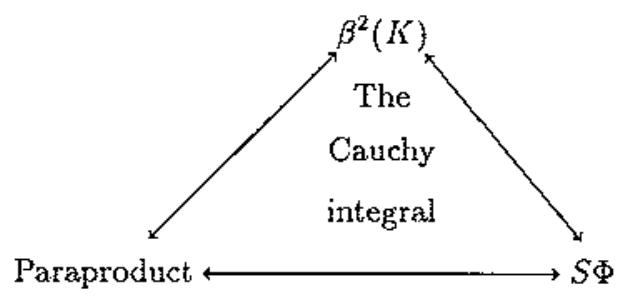

\section{References}

1. C.J. Bishop AND P.W. Jones, Harmonic measure and arclength, Annals of Math. (to appear) (1990).

2. G. DAVID AND S. SEMmES, Article in these proceedings.

3. G. David AND S. SEmmes, Au dela les graphes Lipschitziennes, preprint.

4. J.B. Garneit', P.W. Jones and D.E. Marshale, A Lipschitz decomposition of minimal surfaces, preprint.

5. P.W. JONES, "Square functions, Cauchy integrals, analytic capacity, and harmonic measure," Springer Lecture Notes in Math. 1384, 1989, pp. 24-68.

6. P.W. JONES, Rectifiable sets and the travelling salesman problem, Inventiones Math. (1990). 
7. E.L. LAWLER ET. AL., "The traveling salesman problem," Wiley-Interscience, 1985 .

8. Ch. Pommerenke, On the Green's function of Tuchsian groups, Ann. Acad. Sci. Tenn. 2 (1976), 409-427.

Mathematics Department

Yale University

New Haven CT 06520

U.S.A. 